\title{
Crystallization Behavior and Crystal Morphology of Linear/Long Chain Branching Polypropylene Blends
}

\author{
By Xiang-Dong WANG, ${ }^{1,2}$ Yu-Xia ZHANG, ${ }^{2}$ Ben-Gang LIU, ${ }^{1}$ Zhong-Jie DU, ${ }^{1}$ and Hang-Quan LI ${ }^{1, *}$
}

\begin{abstract}
Strain hardening and crystallization behaviors, crystal structure and morphology of linear/long-chain-branching polypropylene blends (LPP/LCBPP) was studied by means of Rheotens, DSC, XRD, and POM. Upon incorporation of LCBPP into LPP, the melt strength and the strain rate durability of the latter were obviously enhanced, the nucleation and overall crystallization rates were increased and crystallization temperature elevated. At some middle fraction of LCBPP, the most remarkably effects were observed. The content of the branched architecture and the entanglement density were considered responsible for these influences.

KEY WORDS: Long Chain Branching Polypropylene / Linear Polypropylene / Crystallization Behavior / Crystal Morphology /
\end{abstract}

Commodity isotactic PP possesses many desirable physical properties such as good resistance to chemicals, high stiffness, high rigidity, high service temperature, and good temperature stability compared to other polyolefines. However, the lack of melt elasticity strain hardening behavior has limited its applications in extrusion foaming, extrusion coating, blow molding, and thermoforming, where the extensional flow dominates. $^{1-4}$

In order to improve the melt strength of linear isotactic PP (LPP), many efforts were made aiming at enhancing the strain hardening of LPP melt in extensional flow. Crosslinking, broading the molecular weight distribution, blending with other polyolefin were tried in the early researches. ${ }^{5-7}$ Since 1990 s, the introduction of long chain branching onto the backbone of LPP has been considered an effective approach to achieve the high melt strength. ${ }^{8,9}$ A wide variety of methods were employed to achieve the grafting of long-chain side chains onto LPP, including electron-beam irradiation ${ }^{10-13}$ and reactive extrusion with a multifunctional monomer and peroxide. ${ }^{4,14-18}$ Several commercial long chain branching polypropylene (LCBPP) grades were also developed. ${ }^{19}$

However, LCBPP is more expensive than LPP, for this reason, to blend LPP with LCBPP constituted a practical way to generate a PP material with higher melt strength. The LCBPP introduced not only branching points and long side chains, but also more free volumes into the system. How such factors influence the crystallization and melting behavior and the properties of the melt remained interesting problems.

In this work, blends of commercial LPP and LCBPP were prepared and the aforementioned effects were explored.

\section{EXPERIMENTAL}

\section{Materials}

Linear PP (T36F) was obtained from Qilu Petrochemical Co. Ltd. Long chain branching PP (PF814) was purchased from
Basell Co. Ltd. The molecular weight and polydispersity of the PPs were shown in Table I. Antioxidant Irganox B225 was provided by Ciba Specialty Chemicals.

\section{Preparation of the Blends}

Seven blends of T36F/PF814 (LPP/LCBPP) with weight ratios of $100 / 0,90 / 10,80 / 20,70 / 30,60 / 40,50 / 50,0 / 100$ were melt extruded and pelletized using a co-rotating twin screw extruder (WP-30, former WP company, Germany) at $100 \mathrm{rpm}$. The blends were stabilized with $0.2 \mathrm{wt} \%$ Irganox B225. The temperature setup along the barrel of the extruder from the hopper to the die was $175^{\circ} \mathrm{C}, 185^{\circ} \mathrm{C}, 195^{\circ} \mathrm{C}, 205^{\circ} \mathrm{C}$, and $205^{\circ} \mathrm{C}$ (at die).

\section{Analytical Procedures}

Melt strength measurement: The melt strength measurement was carried out on a Göettfert Rheotens melt strength tester (Rheotens71.97, Göttfert, Germany) connected with a single screw laboratory extruder equipped with a capillary die $\left(d_{0}=\right.$ $\left.2 \mathrm{~mm}, L_{0} / d_{0}=15: 1\right)$. The extruding rate was $6.2 \mathrm{~g} / \mathrm{min}$. The drawing accelerating speed of the Rheotens was $20 \mathrm{~mm} / \mathrm{s}^{2}$. The gap of the Rheotens's rollers was $0.9 \mathrm{~mm}$. The distance of the melts subject to drawing was $104 \mathrm{~mm}$.

Thermal analysis: Non-isothermal crystallization characterization was performed using a differential scanning calorimeter (DSC-2C, Perkin-Elmer, USA) in nitrogen atmosphere. Initially, the sample was heated from $40^{\circ} \mathrm{C}$ to $210^{\circ} \mathrm{C}$ at $20^{\circ} \mathrm{C} /$ min and kept for $3 \mathrm{~min}$ at $210^{\circ} \mathrm{C}$ to eliminate the heat history, and subsequently cooled at $20^{\circ} \mathrm{C} / \mathrm{min}$ from $210^{\circ} \mathrm{C}$ to $40^{\circ} \mathrm{C}$ and kept for $5 \mathrm{~min}$ at $40^{\circ} \mathrm{C}$ to observe the crystallization behavior. Finally, the sample was heated again from $40^{\circ} \mathrm{C}$ to $210^{\circ} \mathrm{C}$ at $20^{\circ} \mathrm{C} / \mathrm{min}$ to obverse the melting behavior. In the non-isothermal crystallization characterization, the sample was cooled from $210^{\circ} \mathrm{C}$ to room temperature at $5,10,20$, and $30^{\circ} \mathrm{C} / \mathrm{min}$, respectively. The degree of crystallinity were calculated from the second heating endotherm based on the

\footnotetext{
${ }^{1}$ School of Materials Science and Engineering, Beijing University of Chemical Technology, Beijing 100029, P.R. China

${ }^{2}$ Department of Materials Science and Engineering, Beijing Technology and Business University, Beijing 100037, P.R. China

*To whom correspondence should be addressed (E-mail: lihq@mail.buct.edu.cn).
} 
Table I. Molecular weight and molecular weight distribution of T36F and PF814

\begin{tabular}{lcccc}
\hline PP & $M_{\mathrm{n}}$ & $M_{\mathrm{w}}$ & $M_{\mathrm{z}}$ & $\begin{array}{c}\text { Polydispersity } \\
\text { Index }\end{array}$ \\
\hline T36F & 77000 & 407000 & 1288000 & 5.29 \\
\hline PF814 & 59000 & 523000 & 1792000 & 8.86 \\
\hline
\end{tabular}

Data source: tested by GPC with high temperature at Resin institute of Yanshan Petrochemical Co. Ltd.

heat of fusion of crystallites $(209 \mathrm{~J} / \mathrm{g}$ for $100 \%$ crystalline PP). ${ }^{20}$

Crystal morphology observation: The blend particles were heated to $230{ }^{\circ} \mathrm{C}$ at a heating stove to a melt, which was pressed to a uniform film. The film was soaked in an oil bath of $140^{\circ} \mathrm{C}$ for $2 \mathrm{~h}$, whose flat surface was observed using POM (PM10AD, Olympus, Japan).

X-Ray diffraction: Sample plates for the XRD analysis with a thickness of $2 \mathrm{~mm}$ were molded using an injector (CJ 150 NC, Zhende Plastics Machinery Co. LTD, China). The temperature setup along the barrel of the injection machine from the hopper to the die was $180^{\circ} \mathrm{C}, 195^{\circ} \mathrm{C}$, and $205^{\circ} \mathrm{C}$. The $\mathrm{X}$-ray diffraction experiments were performed using a XRD6000 diffractometer (SHIMADZU, Japan) with $\mathrm{CuK} \alpha$ radiation. The generator was operated at $40 \mathrm{kV}$ and $50 \mathrm{~mA}$. The sample was scanned from $2 \theta=6^{\circ}-36^{\circ}$ at $4^{\circ} / \mathrm{min}$.

\section{RESULTS AND DISCUSSION}

\section{Melt Strength of the Blends}

Although melt strength is an industrial indicator of melt elasticity, it indicates the degree of entanglement in blends of various compositions, and thus helps to explain their crystallization behavior. In Figure 1 the melt strength was plotted against strain rate.

It was well known that LPP possessed poor melt strength because of its linear architecture. The long chain branch in LCBPP, however, imparted the system with high melt strength

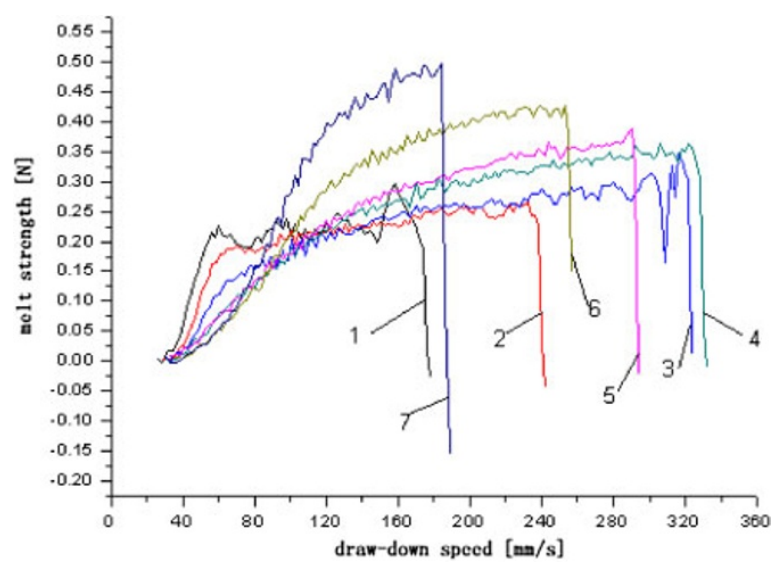

LPP/LCBPP : 1-100/0 2-90/10 3-80/20 4-70/30 5-60/40 6-50/50 7-0/100

Figure 1. Melt strength of LPP/LCBPP blends $\left(190^{\circ} \mathrm{C}\right)$. through strain hardening. Spitael et al. ${ }^{19}$ reported that the blend of LPP/LCBPP exhibited obvious strain hardening even if the loading of LCBPP in the blend was only $10 \mathrm{wt} \%$. Indeed, Figure 1 showed that the higher the content of LCBPP incorporated, the greater the melt strength and the more serious the strain hardening. It was interesting to notice that the strain rate durability of the blends underwent a maximum as the fraction of LCBPP in the blends increased. This could also be attributed to the degree of entanglement in the melt. In de Gennes' reptation model, ${ }^{21}$ a linear chain diffuses along its confining tube in a way analogous to the motion of a snake. However, snake-style reptation is impossible for long-branched polymers because they would have to drag the branches along the tube. Therefore, the branch point of a chain is usually localized in one cell of an entanglement net and the longbranched polymers diffuse by arm retractions, i.e., with octopus-style movement. Obviously, the movement in octopus-style is much slower than that of snake-style, which constitutes the origin of strain-hardening. Linear LPP diffuses with a neat snake-style and no strain-hardening can be observed, When blended with LCBPP, the octopus-style component in movement was introduced, which increases with increasing content of LCBPP. For this reason, as seen in Figure 1, the higher the content of LCBPP, the higher the melt strength. In other hand, the strain rate durability is related to the rate of above mentioned retraction of the long-branches, which is also related to the size of the confining tube. As shown by Rubinstein and Colby, ${ }^{22}$ the diffusion coefficient is proportional to the square of the size of the confining tube determined by the entanglement density. In this work, the molecular weight of LCBPP is smaller than that of LPP, and the length of the branches is even less, as a result, the entanglement density in neat LCBPP system is moderate. Once blended with much longer linear chains, the entanglement density will be remarkably enhanced. For this reason, the diffusion coefficient of the polymer in the blends is determined by two competitive factors, the octopus-style component and the entanglement density. At higher contents of LCBPP, the octopus-like component is low and at lower contents of LCBPP, the entanglement density is low, At middle compositions, the segments acquired the lowest mobility and thus exhibited the largest strain rate durability.

\section{Crystallization Behaviors of the Blends}

Figure 2 shows the DSC cooling traces of LPP/LCBPP blends. Their crystallization parameters were determined according to the Gupta $^{23}$ (Figure 3) and were listed in Table II. The initial slope $S_{i}$ of the crystallization peak characterized the initial nucleating rate. The larger the $S_{i}$, the higher the nucleating rate. The difference between the initial crystallization temperature $T_{\mathrm{c}}$ and the the peak crystallization temperature $T_{\mathrm{p}}\left(T_{\mathrm{c}}-T_{\mathrm{p}}\right)$ represented the total crystallization rate, the less the difference, the higher the total crystallization rate. The width at half height of the crystallization peak $\Delta \mathrm{W}$ represented the distribution of crystal size, the smaller the $\Delta \mathrm{W}$, the narrower the distribution of the crystal size. 


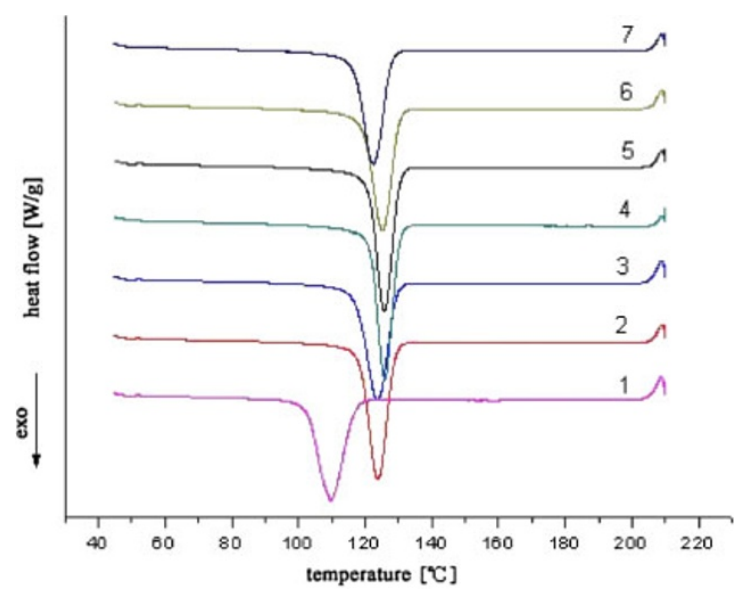

LPP/LCBPP: 1-100/0 2-90/10 3-80/20 4-70/30 5-60/40 6-50/50 7-0/100

Figure 2. DSC cooling behaviors of the seven LPP/LCBPP blends.

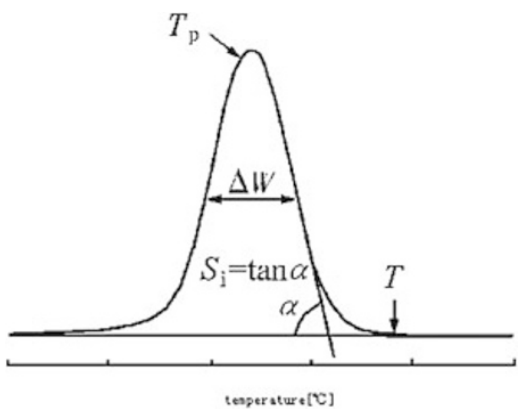

Figure 3. DSC curves illustration of non-isothermal crystallization from Gupta. ${ }^{23}$

One may notice that $T_{\mathrm{c}}, T_{\mathrm{p}}$, and $\mathrm{S}_{\mathrm{i}}$ of neat LCBPP (PF814) were all higher than their counterparts of neat LPP (T36F). It was obviously due to the branched structure of LCBPP, with the branch point acting as the heterogeneous nucleating sites, thus increased both nucleation and crystallization temperatures. However, it was interesting to notice that $T_{\mathrm{c}}, T_{\mathrm{p}}$, and $\mathrm{S}_{\mathrm{i}}$ each exhibited a maximum, $\left(T_{\mathrm{c}}-T_{\mathrm{p}}\right)$ and $\Delta \mathrm{W}$ each a minimum as the weight fraction of LCBPP in the blends increased. In all the maxima and minima, the largest fluctuation was exhibited in the initial slopes $\left(\mathrm{S}_{\mathrm{i}}{ }^{\prime}\right.$ 's), which characterized the initial nucleating rates and was considered here as a determine factor. In the blend systems, the branch points acted as the heterogeneous nucleating sites. However, not all the branch points underwent nucleation, only those with smallest entropy could do so, which was related to the confining effect resulted from both the structure and the environment. Like discussed above, as a result of two competitive factors, octopus-style component and the entanglement density, the systems at middle composition was subjected to the strongest confinement and so did the branch points. As a result, the branch points in middle composition acquired the smallest entropy and thus achieved a largest nucleation rate. This partially explained the maxima in $T_{\mathrm{p}}, T_{\mathrm{c}}$, and the minima in $\left(T_{\mathrm{c}}-T_{\mathrm{p}}\right)$ and $\Delta \mathrm{W}$. In other hand, in systems with higher and lower content of LCBPP, the diffusion rates
Table II. Crystallization parameters of LPP/LCBPP blends (the cooling rate is $20^{\circ} \mathrm{C} / \mathrm{min}$ )

\begin{tabular}{cccccccc}
\hline $\begin{array}{c}\mathrm{T} 36 \mathrm{~F} / \mathrm{P} \\
\mathrm{F} 814\end{array}$ & $T_{\mathrm{p}}\left({ }^{\circ} \mathrm{C}\right)$ & $T_{\mathrm{c}}\left({ }^{\circ} \mathrm{C}\right)$ & $\begin{array}{c}T_{\mathrm{c}}-T_{\mathrm{p}} \\
\left({ }^{\circ} \mathrm{C}\right)\end{array}$ & $\mathrm{S}_{\mathrm{i}}$ & $\boldsymbol{\Delta W}$ & $\begin{array}{c}\Delta \mathrm{H} \\
\left(\mathrm{J} \cdot \mathrm{g}^{-1}\right)\end{array}$ & $\boldsymbol{\alpha}(\%)$ \\
$100 / 0$ & 110 & 117 & 7 & 2.71 & 8.28 & 78.6 & 37 \\
$90 / 10$ & 124 & 129 & 5 & 5.44 & 6.20 & 89.8 & 43 \\
$80 / 20$ & 124 & 129 & 5 & 6.17 & 6.90 & 91.6 & 44 \\
$70 / 30$ & 126 & 130 & 4 & 6.30 & 4.83 & 87.7 & 42 \\
$60 / 40$ & 126 & 130 & 4 & 6.53 & 5.52 & 87.7 & 42 \\
$50 / 50$ & 125 & 130 & 5 & 5.08 & 6.90 & 85.4 & 41 \\
$\mathbf{0} / 100$ & $\mathbf{1 2 3}$ & $\mathbf{1 2 8}$ & $\mathbf{5}$ & $\mathbf{4 . 5 8}$ & $\mathbf{6 . 9 0}$ & $\mathbf{8 1 . 2}$ & $\mathbf{3 9}$ \\
\hline
\end{tabular}

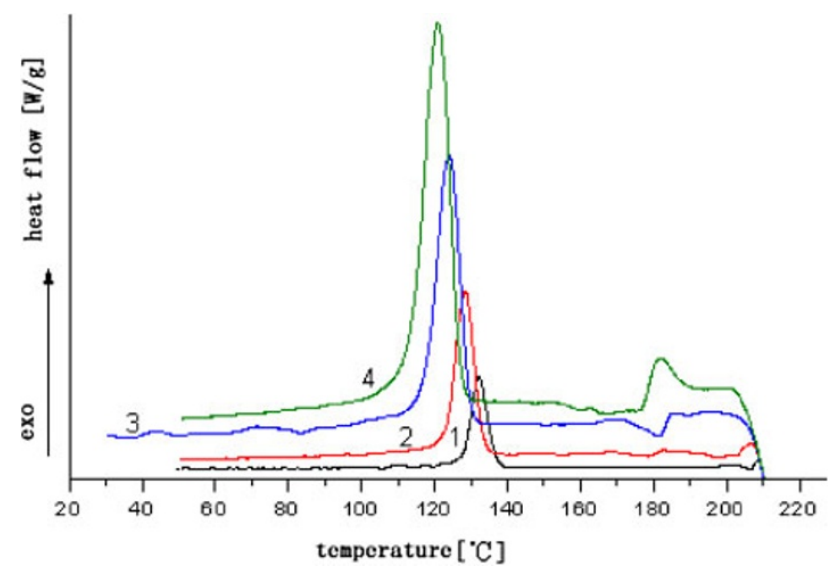

Cooling rates $/{ }^{\circ} \mathrm{C} \cdot \mathrm{min}^{-1}: 1-5 \quad 2-10 \quad 3-20 \quad 4-30$

Figure 4. Crystallization curves of the LPP/LCBPP blend $(80 / 20)$ at various cooling rates.

were larger, which partially compensated their lower nucleation rates, therefore only small fluctuation in values were observed.

Figure 4 presented the effect of cooling rates on the crystallization behavior of a blend with a LPP/LCBPP with ratio of $80 / 20$. It showed that the faster the cooling rate, the lower the $T_{\mathrm{c}}$ and $T_{\mathrm{p}}$. It was naturally attributed to the influence of diffusion. The time-temperature equivalence was well applied to this process. At lower cooling rate, the segments had time long enough to diffuse, the crystallization occurred at a higher temperature; however, at higher cooling rate, the shorter time did not allow the segment to pack and the crystallization appeared at lower temperatures.

\section{Melting Behaviors}

Figure 5 showed the melting behaviors of the blends. The melting points of the blends were listed in Table III. Only one melting point was given for each blend indicating that all the blends are compatible systems. Because of the long chain branches and wide molecular weight distribution of LCBPP, its melting point is lower than that of LPP. In the blends, the higher the fraction of LCBPP, the lower the melting point.

\section{Crystal Structure of the Blends}

Figure 6 shows the typical wide angle X-ray diffraction patterns of the seven blends. All the seven blends have the 


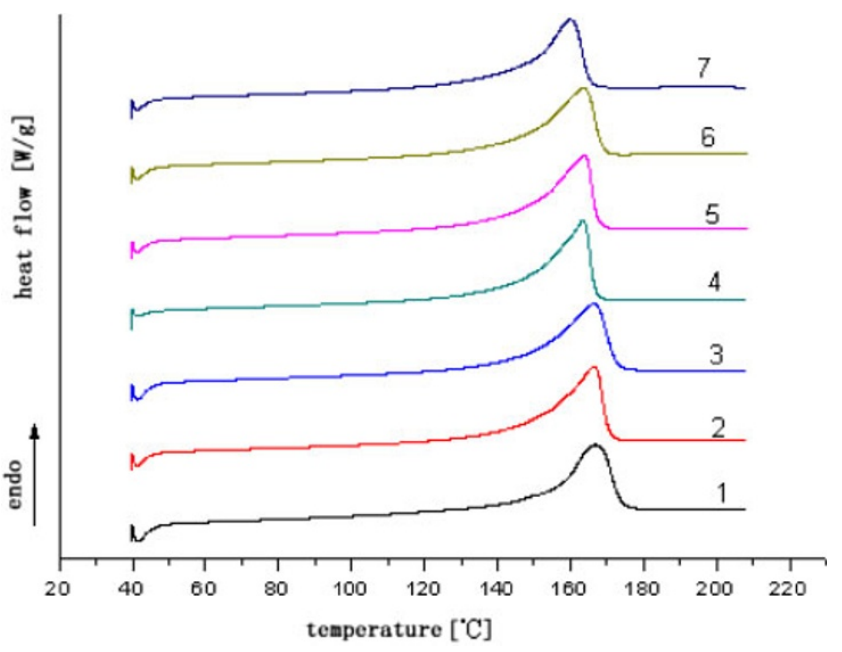

LPP/LCBPP : 1-100/0 2-90/10 3-80/20 4-70/30 5-60/40 6-50/50 7-0/100

Figure 5. Melting curves of the different LPP/LCBPP blends.

Table III. Melting point of LPP/LCBPP blends

\begin{tabular}{cc}
\hline Ratio of LPP over LCBPP & Melting Point $/{ }^{\circ} \mathrm{C}$ \\
\hline $100 / 0$ & 167 \\
$90 / 10$ & 166 \\
$80 / 20$ & 166 \\
$70 / 30$ & 163 \\
$60 / 40$ & 164 \\
$50 / 50$ & 164 \\
\hline $0 / 100$ & 160 \\
\hline
\end{tabular}

similar profiles with very intensive peaks at $2 \theta=14^{\circ}, 17^{\circ}$, $18.5^{\circ}$ and $21.7^{\circ}$, which correspond to the planes of (110), (040), (130), (111), and (131) of $\alpha$ crystal. There is no peak observed corresponding to $\beta$ crystal. This indicates the addition of LCBPP into the blends has not changed the crystal structures.

\section{Crystal Morphology of the Blends}

The Figure 7(a) and 7(g) is for neat LPP and LCBPP, respectively, Figure 7(b) to Figure 7(f) are for blends of the

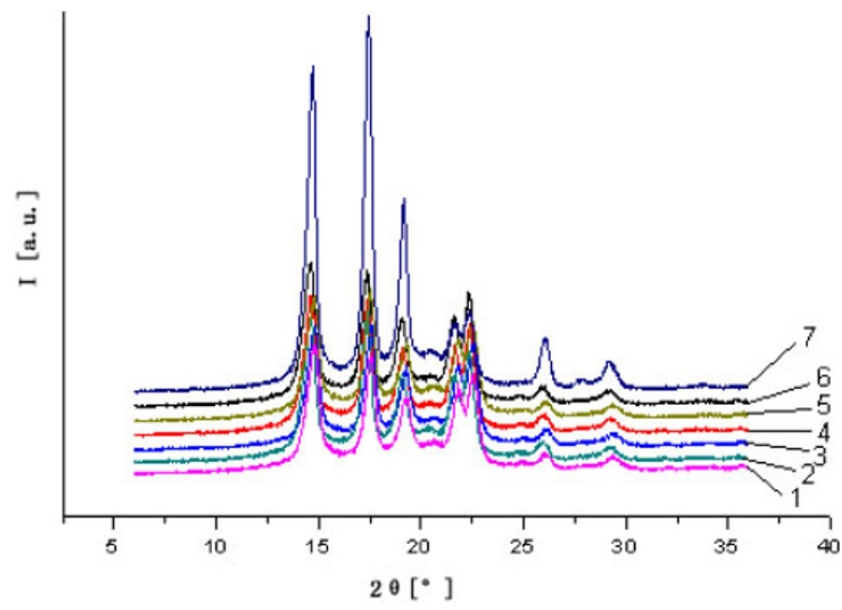

LPP/LCBPP: $1-100 / 0$ 2-90/10 3-80/20 4-70/30 5-60/40 6-50/50 7-0/100

Figure 6. WAXD spectrogram of LPP/LCBPP blends.

two with various compositions. The different morphology was attributed to different mechanism of nucleation. In this work, homogeneous nucleation was assumed to be predominant in the neat LPP, and heterogeneous nucleation induced by the branch points in LCBPP were considered to occur in other case. Being homogeneous nucleated, the nuclei were formed at different time during the crystallization of LPP, and the resultant morphology was irregular Figure 7(a). The Figure 7(b) to Figure $7(\mathrm{~g})$ tell another story. It was noticed that the morphology changed from fine to coarse and to fine again. This was a result of the compromise of the rates of nucleation and diffusion. For the middle composition ( $\mathrm{d}$ and $\mathrm{e}$ ), where the nucleation rate was higher and diffusion rate was lower, the growth of the crystallites is predominantly surrounding the nuclei, few segments were organized in the gaps leaving a coarse morphology. In other hand, in systems with lower or higher LCBPP contents (Figure 7b, c, f, and g), the nucleation rate was lower and diffusion rate was higher, the segments could easily fill the gap among crystallites and where voids occurred, resulting in a more uniform structure, consequently fine morphologies were observed.

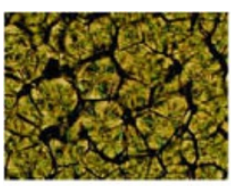

(a)

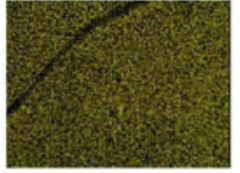

(b)

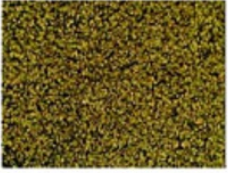

(c)

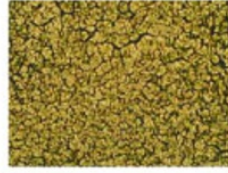

(d)

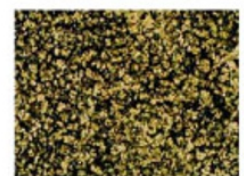

(e)

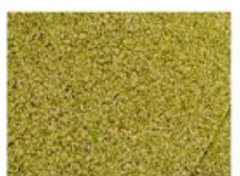

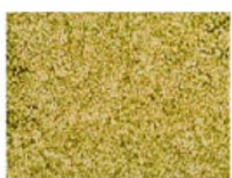

(g)

LPP/LCBPP : (a)100/0 (b)90/10 (c)80/20 (d)70/30 (e)60/40 (f)50/50 (g) 0/100

Figure 7. Polariscope photos of different LPP/LCBPP blends. 


\section{CONCLUSIONS}

When LCBPP was incorporated into LPP, an octopus-style component of movement was introduced in addition to original snake-style, which resulted in high melt strength. The entanglement density among the segments of LPP and LCBPP determined both the nucleation and diffusion rates of the polymers, which in turn influence the strain hardening and crystallization behaviors. At certain middle content of LCBPP, the blends acquired the lowest diffusion rates, and the widest strain rate durability, highest nucleation and overall crystallization rates could be observed. The inclusion of LCBPP did not change the crystal form.

Acknowledgment. Financial assistance from Beijing Foundation of Natural Science (Grant Number: 2062008) is gratefully acknowledged.

Received: November 1, 2007

Accepted: January 31, 2008

Published: March 19, 2008

\section{REFERENCES}

1. C. B. Park and L. K. Cheung, Polym. Eng. Sci., 37, 1 (1997).

2. H. E. Naguib, C. B. Park, and N. Reichelt, J. Appl. Polym. Sci., 91, 2661 (2004).

3. G. J. Nam, J. H. Yoo, and J. W. Lee, J. Appl. Polym. Sci., 96, 1793 (2005).

4. A. D. Gotsis, L. F. Zeevenhoven, and A. H. Hogt, Polym. Eng. Sci.,
44, 973 (2004).

5. J. Alteepping and J. P. Nebe, U. S. Patent 4,940,736 (1990).

6. A. Nojiri, T. Sawasaki, and T. Koreeda, U. S. Patent 4,424,293 (1984).

7. Y. D. Lee and L. F. Wang, J. Appl. Polym. Sci., 32, 4639 (1986).

8. S. B. Joseph, M. W. John, Jr. DeNicola, and J. Anthony, U. S. Patent 4,916,198 (1990).

9. Jr. DeNicola and J. Anthony, Process for making a propylene polymer with free-end long chain branching and use thereof. U. S. Patent 5,047,485 (1991).

10. B. Krause, L. Häußler, and V. Dieter, J. Appl. Polym. Sci., 100, 634 (2006).

11. B. Krause, M. Stephan, S. Volkland, D. Voigt, L. Häußler, and H. Dorschner, J. Appl. Polym. Sci., 99, 260 (2006).

12. B. Krause, D. Voigt, L. Häußler, D. Auhl, and H. Münstedt, J. Appl. Polym. Sci., 100, 2770 (2006).

13. T. Zaharescu, R. Setnescu, S. Jipa, and T. Setnescu, J. Appl. Polym. Sci., 77, 982 (2000).

14. J. Saito, S. Kawazoe, and S. Kikukawa, U. S. Patent 5,416,169 (1995).

15. A. H. Hogt, O. B. Ho, and H. Westmijze, Patent WO. 97/49759 (1996).

16. A. H. Hogt, B. Fischer, and G. K. Spijkerman, Patent WO. 99/27007 (1999).

17. Xiaochuan Wang, C. Tzoganakis, and G. L. Rempel, J. Appl. Polym. Sci., 61, 1395 (1996).

18. M. Sugimoto, T. Tanaka, Y. Masubuchi, and J. Takimoto, J. Appl. Polym. Sci., 73, 1493 (1999).

19. P. Spitael and C. W. Macosko, Polym. Eng. Sci., 44, 2090 (2004).

20. "Polymer Handbook," J. Brandrup, E. H. Immergut, and E. A. Grulke, Ed., Wiley, New York, 1998.

21. "Introduction to Polymer Dynamics," P. G. D. Gennes, Ed., Cambridge University Press, UK, 1990.

22. "Polymer Physics," M. Rubinstein and R. H. Colby, Ed., Oxford University Press, UK, 2003.

23. A. K. Gupta and S. N. Purwar, J. Appl. Polym. Sci., 29, 1595 (1984). 